\title{
Thermal Rendering Based on Thermal Diffusion Equation
}

\author{
Yukiko Osawa* Member, Seiichiro Katsura* Senior Member
}

(Manuscript received July 12, 2018, revised April 17, 2019)

\begin{abstract}
Actuator arrays that consist of a planar arrangement of actuators are widely used for transportation of objects or shaping a rough surface by controlling the actuators cooperatively. In particular, thermal displays that consist of multiple heat sources spread over a surface have been developed for many years. Generally, the expression ability is determined by the number of actuators in actuator arrays, and accordingly, thermal displays require many heat sources to ensure a high spatial resolution of temperature distribution. However, the number of usable heat sources is limited because of mechanical issues. In order to solve this problem, a control method that employs a model derived from a thermal diffusion equation and is used between two heat sources, is proposed in this paper. By using the proposed method, any selected point of the heat conduction can be expressed using only one heat source. Therefore, infinite number of nodes of propagation can be reproduced by selecting a desired node. The method is expected to extend the technology for controlling multiple actuators or heat sources on a surface.
\end{abstract}

Keywords: actuator array, thermal rendering, heat conduction control, thermal diffusion equation, temperature distribution, peltier device

\section{Introduction}

Actuator arrays that consist of a planar arrangement of actuators are widely used for transportation of objects or shaping a rough surface by controlling the actuators cooperatively. For example, multiple actuators are used for spatial haptic motion $^{(1)(2)}$. In particular, thermal displays that consist of multiple heat sources spread over a surface have been developed for many years.

In many cases, Peltier devices ${ }^{(3)-(5)}$ are used as heat sources for thermal displays. The device is useful because it can be controlled to be hot or cool. There are a lot of studies about the device itself, such as robust temperature control ${ }^{(6)}$ or heat flow control ${ }^{(7)}$. Both temperature and heat flow can be controlled using some control method ${ }^{(8)-(10)}$.

Controlling multiple heat sources as thermal displays have also been studied. The study ${ }^{(11)(12)}$ developed thermal display by spreading some Peltier devices. In addition, 4-channel and 12-channel dynamic thermal displays that were used to create a continuous cooling sensation without changing the average temperature is proposed ${ }^{(13)}$. Besides, space-time interactions of thermal stimuli is presented ${ }^{(14)}$. Some studies focus on a real heat conduction on material, and some control methods are proposed ${ }^{(15)}$. The control system of these conventional methods are for single Peltier device, and temperature distribution can not be controlled at once. Therefore, these thermal displays require many heat sources to render a high spatial resolution of temperature distribution.

Heat conduction can be controlled virtually by setting the virtual thermal conductance based on a lumped parameter model ${ }^{(16)}$. Although, heat conduction has to be treated as a distributed parameter system considering both temporal and

\footnotetext{
* Department of System Design Engineering, Keio University 3-14-1, Hiyoshi, Kohoku-ku, Yokohama 223-8522, Japan
}

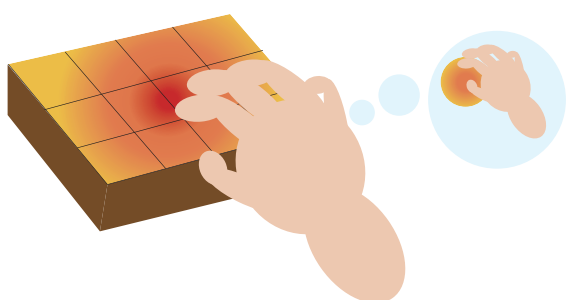

Fig. 1. The concept figure of this paper

spatial information. There are some studies that control thermal system using a thermal diffusion equation ${ }^{(17)-(19)}$. Most of these studies treat the heat phenomenon as an integrator and time delay element using approximation. In many cases, these models are used for only monitoring, not controlling. However, there is a possibility that such a model can be used for thermal displays.

Generally, the expression ability of thermal system is determined by the number of heat sources. However, the number of usable heat sources is limited because of mechanical issues. In order to solve this problem, a control method that employs a model derived from a thermal diffusion equation and is used between two heat sources, is proposed in this paper. The objective of this paper is shown in Fig. 1. By using the proposed method, freely-selected point of the heat conduction can be expressed by only one heat source. Therefore, infinite number of nodes of heat conduction can be reproduced by selecting a desired node. The method is expected to extend the technology for controlling multiple actuators or heat sources on a surface. Some experiments and simulations are conducted to confirm the proposed method.

\section{Advantage of the Proposed Method}

Advantage of the proposed method is rendering ability of single actuator can be improved. In general, functionality 
of control system is determined by the number of actuators involved the system. As for thermal display, a lot of heat sources are needed to render a precise thermal sensation. However, control system becomes large by an amount equal to the number of heat sources, and the number of usable heat sources is limited. This paper focuses on this point. In the proposed method, command of each heat source includes thermal dynamics of all positions. Therefore, it is possible to render a freely selected point of heat conduction and the position of rendering point can be changed as needed by using the proposed method. Accordingly, expressed resolution can be improved by using a limited number of heat sources. The method can be applied to every system of actuators array, not only thermal display.

\section{Modeling of the Thermal System}

3.1 Precondition of the Thermal System There are some preconditions for modeling in this paper. As for a whole thermal system,

- Multiple heat sources are arranged in a line.

- Real heat interference between heat sources are not occurred.

- Virtual heat conduction is controlled between heat sources.

- One heat source is treated as single point.

(The size of the heat source is not considered in this model).

Here, heat interference between heat sources are not considered in the thermal model because each heat source is compensated from a heat disturbance observer (HDOB) ${ }^{(6)}$ that can reject disturbance such as heat interference. As for virtual heat conduction,

- One-dimensional heat conduction is considered.

- The law of the conservation of energy (first law of thermodynamics) is established.

- The Fourier's law (second law of thermodynamics) is established.

- Radiation of heat is not considered.

- Thermal parameter of heat conduction is uniform.

The assumption is related to the heat flow command of the Peltier device. Therefore, these assumptions influence on the temperature responses of the device.

3.2 Composition of the Thermal System The thermal system used in this paper consists of multiple heat sources, called Peltier devices. The array of heat sources are called 'thermal display' in this paper. Thermal phenomena in the thermal display is modeled by using thermal network $\operatorname{method}^{(20)(21)}$. The model is shown in Fig. 2. Here, $C_{\mathrm{v}}, R_{\mathrm{v}}$, $C_{\mathrm{p}}, R_{\mathrm{p}}, R_{\mathrm{e}}, \alpha, I, T_{\mathrm{a}}, T_{\mathrm{p}}$, and $T_{\mathrm{pb}}$ stand for virtual thermal conductance, virtual thermal resistance, the one of the Peltier device, electric resistance, Seebeck coefficient, current, absolute temperature, and temperature of both sides of Peltier device, respectively. In the case, heat conduction between two Peltier device is virtual one. By using the model, heat conduction can be expressed by using single heat source. The proposed method needs some heat sources using for setting the boundary conditions of virtual heat conduction and rendering it. As for the experiments in this paper, one or two devices were used for boundary conditions of virtual heat conduction, and two or three devices were used for rendering the

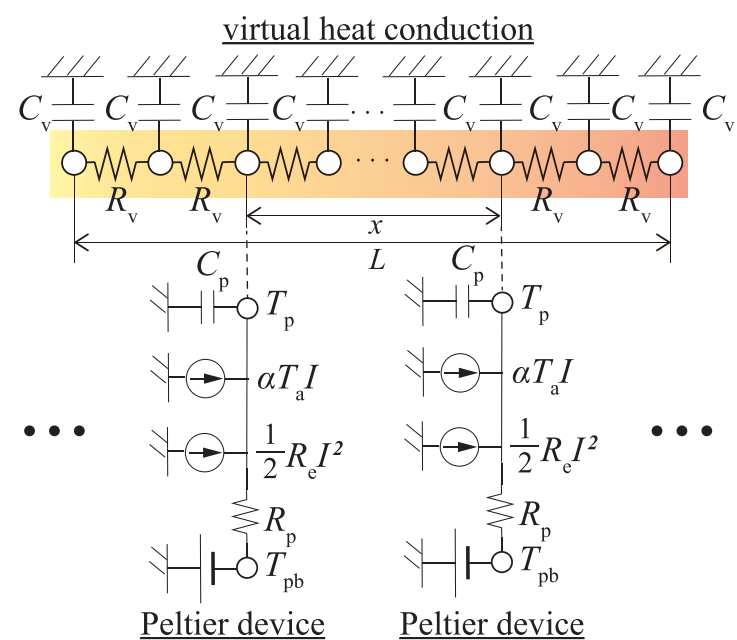

Fig. 2. Modeling of thermal system using a thermal network method

virtual heat conduction.

3.3 Modeling of Heat Conduction By using the precondition mentioned in Section 2.1, virtual heat conduction between two heat sources are modeled based on a thermal diffusion equation. In this paper, heat flow dimensional equation is used because derived model of heat conduction becomes simple one. The equation is expressed as

$$
\begin{aligned}
& \frac{\partial q(t, x)}{\partial x}=c \frac{\partial^{0.5} q(t, x)}{\partial^{0.5} t} . \\
& c=\sqrt{\frac{c_{\mathrm{s}} \rho}{\lambda}}=\sqrt{\frac{1}{c_{\mathrm{d}}}}, \cdots
\end{aligned}
$$

where $t, x, q, c_{\mathrm{s}}, \rho, \lambda$, and $c_{\mathrm{d}}$ stand for time, space, heat flow, specific heat, density, and thermal diffusivity, respectively. Here, relationship between temperature and heat flow ${ }^{(22)(23)}$ can be calculated as

$$
\begin{aligned}
q(t, x) & =c \frac{\partial^{0.5}}{\partial t^{0.5}} T(t, x) \ldots \ldots \ldots \ldots \ldots \ldots \ldots \ldots \ldots \ldots \ldots \ldots \ldots \ldots \ldots \ldots \ldots \ldots
\end{aligned}
$$

where $T$ denotes temperature. The initial condition is set as

$$
q(0, x)=0
$$

By using (5), the solution of (1) is derived as

$$
q(s, x)=e^{-c x \sqrt{s}} q(s, 0)
$$

where $s$ denotes the Laplace operator. The block diagram of the fundamental model is shown in Fig. 3. In the case of Dirichlet boundary condition, heat flow from both sides of heat sources can be calculated as

$$
q(s, x)=e^{-c x \sqrt{s}} q(s, 0)+e^{-(L-x) c \sqrt{s}} q(s, L) . \cdots \cdots \cdots(7)
$$

Next, frequency characteristics of the thermal model is analyzed. $e^{-\sqrt{s}}$ can be rewritten in a polar coordinate system shown as

$$
e^{-\sqrt{s}}=e^{-\sqrt{j \omega}}=e^{-\sqrt{j} \sqrt{\omega}}
$$

By using Euler's formula, $\sqrt{j}$ is shown as 


$$
\stackrel{q(s, 0)}{\longrightarrow} e^{-c x \sqrt{s}} \stackrel{q(s, x)}{\longrightarrow}
$$

Fig. 3. Block diagram of heat conduction based on a thermal diffusion equation

$$
\begin{aligned}
\sqrt{j} & =j^{\frac{1}{2}} \\
& =\left(e^{j \frac{\pi}{2}}\right)^{\frac{1}{2}}=e^{j \frac{\pi}{4}} \\
& =\cos \frac{\pi}{4}+j \sin \frac{\pi}{4} \\
& =\frac{1}{\sqrt{2}}+j \frac{1}{\sqrt{2}} \cdots
\end{aligned}
$$

Therefore, the thermal model derived from (8) is calculated as

$$
\begin{aligned}
e^{-\sqrt{j} \sqrt{\omega}} & =e^{-\left(\frac{1}{\sqrt{2}}+j \frac{1}{\sqrt{2}}\right) \sqrt{\omega}} \\
& =e^{-\left(\sqrt{\frac{\omega}{2}}+j \sqrt{\frac{\omega}{2}}\right)} \\
& =e^{-\sqrt{\frac{\omega}{2}}} e^{-\sqrt{\frac{\omega}{2}} j} .
\end{aligned}
$$

From (10), the thermal model has a damping element and a delay element. The model are used for heat flow command of each heat source. Implementation of the model is explained in the next section.

3.4 Implementation of Distributed Parameter Model In order to implement the model derived in previous chapter, the number of terms are reduced by an approximation method. $e^{-\sqrt{T s}}$ can be approximated using power series expansion shown as

$$
e^{-\sqrt{T s}}=\frac{1}{e^{\sqrt{T s}}} \approx \frac{1}{\frac{T}{2} s+\sqrt{T s}+1} .
$$

In this paper, Oustaloup's filter ${ }^{(22)(23)}$ is used to implement (7). The definition of the filter is shown as

$$
\begin{aligned}
& s^{\gamma} \approx K \prod_{\mathrm{k}=-\mathrm{N}}^{\mathrm{N}} \frac{s+\omega_{\mathrm{k}}^{\prime}}{s+\omega_{\mathrm{k}}}(k=-N, \ldots, N) \cdots \ldots \ldots \ldots \\
& \omega_{\mathrm{k}}^{\prime}=\omega_{\mathrm{b}}\left(\frac{\omega_{\mathrm{h}}}{\omega_{\mathrm{b}}}\right)^{\frac{k+N+(1-\gamma) / 2}{2 N+1}} \\
& \omega_{\mathrm{k}}=\omega_{\mathrm{b}}\left(\frac{\omega_{\mathrm{h}}}{\omega_{\mathrm{b}}}\right)^{\frac{k+N+(1+\gamma) / 2}{2 N+1}} \\
& K=\left(\frac{\omega_{\mathrm{h}}}{\omega_{\mathrm{b}}}\right)^{-\frac{\gamma}{2}} \prod_{\mathrm{k}=-\mathrm{N}}^{\mathrm{N}} \frac{\omega_{\mathrm{k}}}{\omega_{\mathrm{k}}^{\prime}},
\end{aligned}
$$

where $\gamma, N, \omega_{\mathrm{b}}$, and $\omega_{\mathrm{h}}$ stand for order of the Laplace operator, order of the filter, low frequency limit, and upper frequency limit, respectively. Figure 4 shows bode diagram of the approximation model comparing to the theoretical model. In this case, $x$ is set to $15 \mathrm{~mm}$, and the thermal diffusivity of copper is used for calculation. $\omega_{\mathrm{b}}$ and $\omega_{\mathrm{h}}$ were set to $10^{-2} \mathrm{rad} / \mathrm{s}$ and $10^{2} \mathrm{rad} / \mathrm{s}$, respectively. The transfer function of the thermal model after approximation is shown as

$$
\begin{aligned}
\frac{q(s, x)}{q(s, 0)} & =e^{-c x \sqrt{s}} \\
& =\frac{0.2921 s+0.004021}{s^{2}+0.3755 s+0.004203}
\end{aligned}
$$

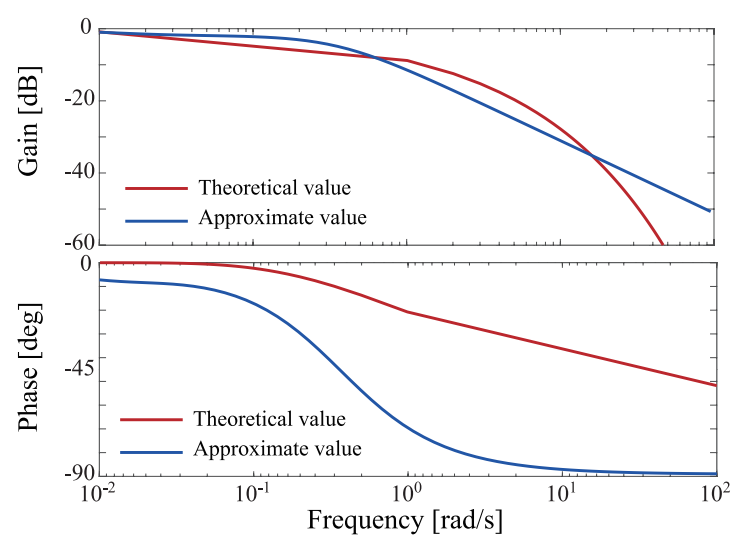

Fig. 4. Bode diagram of approximated model comparing to the theoretical value

$$
\begin{aligned}
& =\frac{0.2903}{s+0.3640}+\frac{0.0018}{s+0.0115} \\
& =\frac{B}{s+A}+\frac{D}{s+C} . \cdots \cdots \cdots
\end{aligned}
$$

Here, $N$ is set to 2 , because it is enough to verify the proposed method within the bandwidth between $10^{-2} \mathrm{rad} / \mathrm{s}$ and $10^{2} \mathrm{rad} / \mathrm{s}$. In order to increase resolution of the proposed method, order of the filter has to be increased.

3.5 Modeling of Peltier Device Peltier devices are used as a heat source in this method. The Peltier devices are thermoelectric elements and used for heating/cooling surface on the thermal display. It generates heat based on Peltier effect, and reference value of the heat flow generated from the device is described as

$$
q^{\mathrm{ref}}=-\alpha T_{\mathrm{a}} I^{\mathrm{ref}}
$$

where subscript ref stands for reference value of Peltier device. Therefore, Peltier device can be controlled by a current.

\section{Control Algorithm of the Heat Conduction System}

4.1 Control at Selected Point of Heat Conduction The whole block diagram of the control system is shown in Fig. 5. Subscript $1, \mathrm{r}, \mathrm{x}, K_{\mathrm{pt}}, K_{\mathrm{dt}}, K_{\mathrm{ph}}, K_{\mathrm{dh}}$, subscript $\mathrm{cmd}$, cmp, dis, and res stand for the left, right side, position $x$, and proportional and differential gain of temperature and heat flow control, command value, compensated value, disturbance, and response, respectively. The green area in Fig. 5 is the model of the device using for setting boundary condition of virtual heat conduction. The temperature of the device is controlled by proportional and differential controller. The yellow area in Fig. 5 is the model of the device using for rendering virtual heat conduction. The device is controlled based on the command at red area in Fig. 5. Here, the Peltier devices are controlled robustly by the HDOB that to remove disturbance factors such as unnecessary heat flow generated into the control system. The command value of the device for rendering virtual heat conduction will be explained in the next section.

4.2 Virtual Heat Conduction Between Heat Sources The command of heat flow is calculated as

$$
q^{\mathrm{cmd}}=e^{-c x \sqrt{s}} q_{1}+e^{-(L-x) c \sqrt{s}} q_{\mathrm{r}}
$$




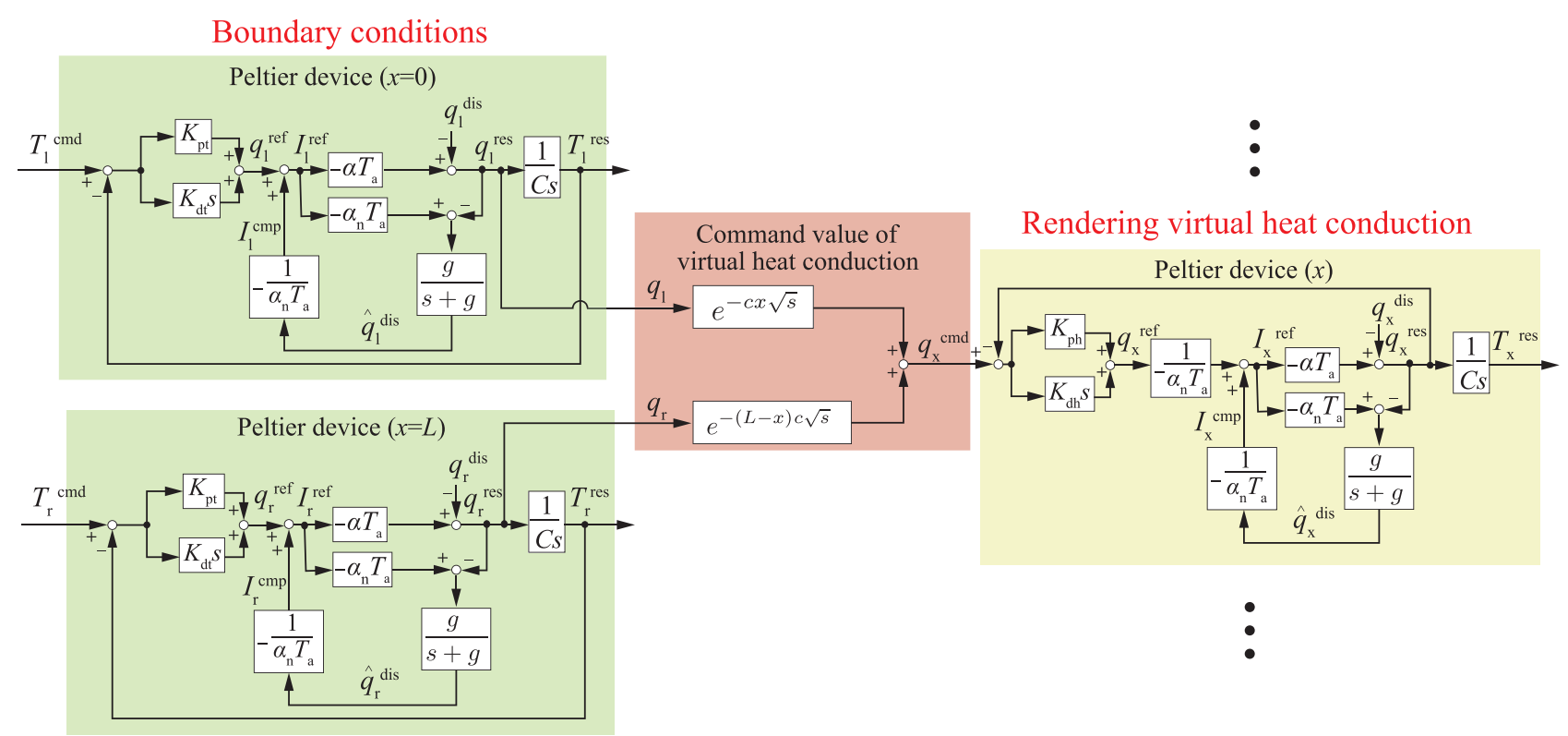

Fig. 5. Block diagram of the whole control system

where $q_{1}$ and $q_{\mathrm{r}}$ stand for heat flow of the boundary heat sources. From (18), heat flow command is changed depending on $x$. Therefore, heat flow at selected point can be expressed using heat conduction from $q_{1}$ and $q_{\mathrm{r}}$. The command is used for controlling Peltier device as if any point of heat conduction can be rendered. The devices for rendering heat conduction are controlled by using the heat flow command derived from (18). Heat flow of these devices are controlled with compensation from a disturbance observer. The actual plant model at the $x$ is derived as

$$
\begin{aligned}
q_{\mathrm{x}}^{\mathrm{ref}}-q^{\mathrm{dis}} & =q_{\mathrm{x}}^{\mathrm{res}} \ldots \\
& =C s T_{\mathrm{x}}^{\mathrm{res}} .
\end{aligned}
$$

Here, the reference value of the device for rendering virtual heat conduction is expressed as

$$
q^{\mathrm{ref}}=\left(K_{\mathrm{ph}}+K_{\mathrm{dh}}\right)\left(q^{\mathrm{cmd}}-q^{\mathrm{res}}\right) .
$$

In the next section, some experiments are conducted to verify the validity of the proposed method.

\section{Experiment}

5.1 Outline of Experiments Figure 6 shows the experimental setup of virtual heat conduction system. There are four Peltier devices that are spread on the same surface of heat sink, and four thermocouples and heat flux sensors. The device number are set, such as devices $1,2,3$, and 4, respectively. The size of the Peltier devices are $20 \mathrm{~mm} \times 20 \mathrm{~mm}$. Thermal parameters of the virtual heat conduction were set based on a copper material that the length $(L)$ is $45 \mathrm{~mm}$. The Peltier devices are used as actuators and they reproduced virtual heat conduction $(x)$ and its boundary condition $(x=0, L)$. A temperature sensors (thermocouples and heat flux sensors) were attached to each device and get temperature and heat flow information of the Peltier device. The command of $q_{\mathrm{x}}$ is derived from $q_{1}(x=0)$ and $q_{\mathrm{r}}(x=L)$, and proportional and differential controllers were used for heat flow control based on the command. Here, the relationship between the experimental set up and the proposed control system is shown in

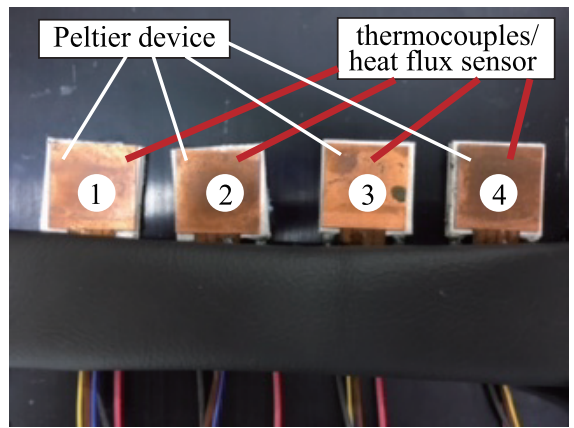

Fig. 6. Experimental setup of virtual heat conduction system
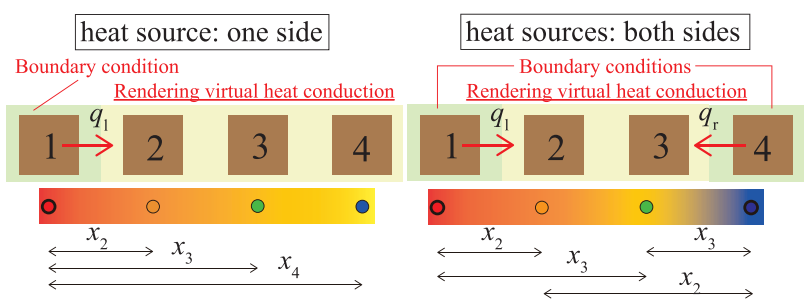

Fig. 7. The relationship between the experimental set up and the proposed control system

Fig. 7, where $x$ stands for the distance from the heat source. When virtual heat conduction from one side of heat source is reproduced, the device 1 in Fig. 6 is used as a boundary condition and devices 2,3 , and 4 reproduce the virtual heat conduction. This means that temperature of the device 1 is controlled and the other devices are controlled to the command based on a thermal diffusion equation including the heat flow response of the device $1\left(q_{1}\right)$. In this experiment, $q_{1}$ was derived from temperature of the device $1\left(T_{1}\right)$ that was controlled to $1 \mathrm{~K}$, and $q_{\mathrm{r}}$ was 0 . When virtual heat conduction from both sides of heat source is reproduced, devices 1 and 4 are used as boundary conditions and devices 2 and 3 reproduces virtual heat conduction. Here, temperatures of devices 1 and 4 are controlled to $1 \mathrm{~K}$ and $-1 \mathrm{~K}$, and $q_{1}$ and $q_{\mathrm{r}}$ 


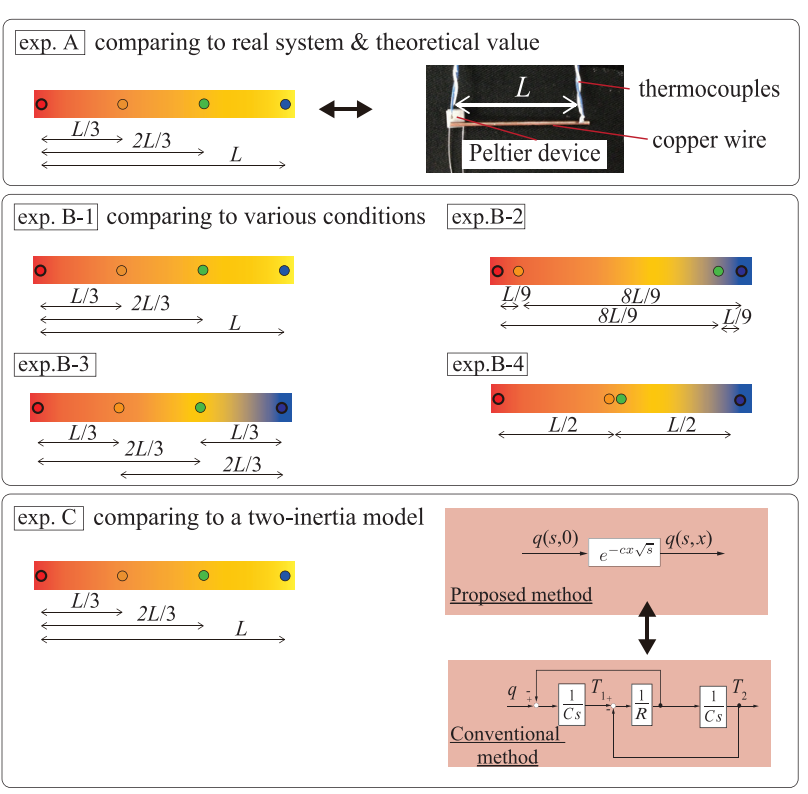

Fig. 8. Outline of the experiments

are derived from these temperatures.

There are three kinds of experiments. The outline of the experiments are shown in Fig. 8. First, implemented heatconduction model was verified. In case of exp. A, the left side of the device (device 1) was controlled to $1 \mathrm{~K}$, and the command of the right side of it (device 2) were monitored. The command of heat flow was compared to the real heat conduction using a copper wire, that is attached heat source at the left side of the wire. The length of the wire is also $45 \mathrm{~mm}$. The experimental results were also compared to simulation results that are derived from the theoretical value at a selected point of virtual heat-conduction material. In addition, frequency characteristics between response of heat flow and the command of heat flow derived from it were verified. Here, sweep signal was applied for 100 seconds, the frequency was changed from $0.01 \mathrm{rad} / \mathrm{s}$.

Second, heat-conduction control was verified. In case of exp. B-1, temperature of left side of the device (device 1) was controlled to $1 \mathrm{~K}$. The devices 2,3 , and 4 were controlled from the command of heat flow based on (18). In this experiments, $x$ was set to $15 \mathrm{~mm}(L / 3), 30 \mathrm{~mm}(2 L / 3)$, and $45 \mathrm{~mm}$ (L) (exp. B-1). In exps. B-2, B-3, and B-4, temperature of both sides of the devices (device 1, device 4) were controlled to $1 \mathrm{~K}$ and $-1 \mathrm{~K}$. As for another devices, $x$ used for the command of heat flow is changed. In case of exp. B-2, the command of devices 2 and 3 were set to $5 \mathrm{~mm}(L / 9)$ and $40 \mathrm{~mm}$ $(8 L / 9)$ from the left. In case of exp. B-3, the command of devices 2 and 3 were set to $15 \mathrm{~mm}(L / 3)$ and $30 \mathrm{~mm}(2 L / 3)$ from the left. In case of exp. B-4, the command of devices 2 and 3 were set to $22.5 \mathrm{~mm}(L / 2)$.

Third, the proposed method was compared to a lumped parameter model. In case of exp. C, temperature response using a distributed parameter model when $x$ was set to $15 \mathrm{~mm}$ was compared to the one based on a lumped parameter model. In this case, virtual heat conduction based on a two-inertia model was used as a conventional method. The role of the devices in the experiments are shown in Table 1. Here, "B" and "V" mean that the device is used for rendering boundary
Table 1. The role of the devices in the experiments

\begin{tabular}{c|c|c|c|c}
\hline & device 1 & device 2 & device 3 & device 4 \\
\hline exp. A & $\mathrm{B}$ & $\mathrm{V}$ & $\mathrm{V}$ & $\mathrm{V}$ \\
exp. B-1 & $\mathrm{B}$ & $\mathrm{V}$ & $\mathrm{V}$ & $\mathrm{V}$ \\
exp. B-2 & $\mathrm{B}$ & $\mathrm{V}$ & $\mathrm{V}$ & $\mathrm{B}$ \\
exp. B-3 & $\mathrm{B}$ & $\mathrm{V}$ & $\mathrm{V}$ & $\mathrm{B}$ \\
exp. B-4 & $\mathrm{B}$ & $\mathrm{V}$ & $\mathrm{V}$ & $\mathrm{B}$ \\
exp. C & $\mathrm{B}$ & $\mathrm{V}$ & $\mathrm{V}$ & $\mathrm{V}$ \\
\hline
\end{tabular}

Table 2. Parameter values of experiments

\begin{tabular}{c|c|c}
\hline Parameter & Description & Value \\
\hline \hline$K_{\mathrm{pt}}$ & Proportional gain for temperature control & 1.0 \\
$K_{\mathrm{dt}}$ & Differential gain for temperature control & 2.0 \\
$K_{\mathrm{ph}}$ & Proportional gain for heat flow control & 7.0 \\
$K_{\mathrm{dh}}$ & Differential gain for heat flow control & 3.74 \\
$\alpha_{\mathrm{n}}$ & Nominal Seebeck coefficient & $0.023 \mathrm{~V} / \mathrm{K}$ \\
$C_{\mathrm{n}}$ & Nominal thermal capacitance & $2.0 \mathrm{~J} / \mathrm{K}$ \\
$g_{\mathrm{d}}$ & Cut-off frequency for pseudo differential & $1.0 \mathrm{~Hz}$ \\
$g_{\mathrm{i}}$ & Cut-off frequency for HDOB & $1.0 \mathrm{~Hz}$ \\
$L$ & Length of virtual conduction material & $45 \mathrm{~mm}$ \\
$c$ & Thermal diffusivity (simulation) & $95.34 \mathrm{~mm} / \mathrm{s}$ \\
$A$ & Equation (16) & $0.3640(x=15 \mathrm{~mm})$ \\
& & $0.1305(x=30 \mathrm{~mm})$ \\
& & $0.0778(x=45 \mathrm{~mm})$ \\
$B$ & Equation (16) & $0.2903(x=15 \mathrm{~mm})$ \\
& & $0.0909(x=30 \mathrm{~mm})$ \\
& & $0.0473(x=45 \mathrm{~mm})$ \\
$C$ & Equation (16) & $0.0115(x=15 \mathrm{~mm})$ \\
& & $0.0079(x=30 \mathrm{~mm})$ \\
& & $0.0067(x=45 \mathrm{~mm})$ \\
$D$ & Equation (16) & $0.0018(x=15 \mathrm{~mm})$ \\
& & $0.0017(x=30 \mathrm{~mm})$ \\
& & $0.0018(x=45 \mathrm{~mm})$ \\
\hline
\end{tabular}

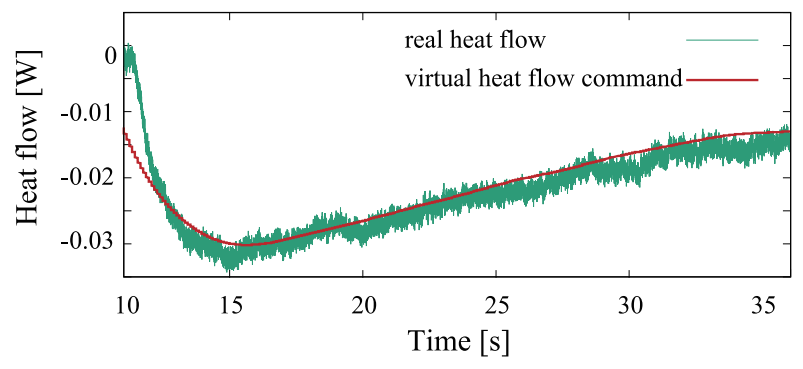

(a) Experimental results comparing to real heat conduction.

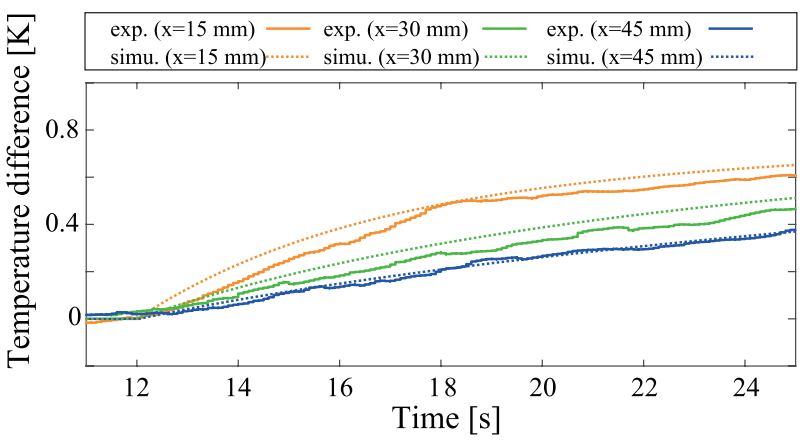

(b) Transient responses comparing to theoretical value.

Fig. 9. Experimental results of exp. A

condition (temperature control) and rendering virtual heat conduction (heat flow control), respectively. The parameter values used for the experiment are shown in Table 2.

\subsection{Experimental Results}

5.2.1 Verification of Implemented Heat-Conduction Model Figure 9 (a) shows heat flow command of virtual heat conduction and the responses of real heat conduction in case of exp. A. The response of heat flow was calculated from 


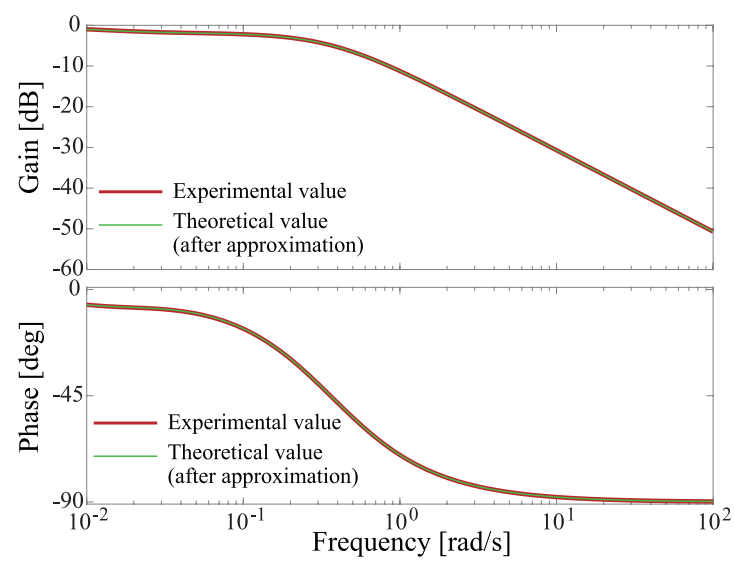

Fig. 10. Bode diagram of heat flow comparing experimental results and theoretical value

temperature response of thermocouples attached to the wire. Here, the temperature control for real heat conduction was started in 10 seconds, and the data plotted on the graph after 10 seconds. The temperature control of heat sourc e for the virtual heat conduction was also started from 10 seconds, and the initial value of the heat flow responses were different because calculation of heat flow command for virtual heat conduction was started in 0 seconds. From Fig. 9 (a), the command of heat flow is almost same with the real one. Thus, it can be said that the command of heat flow derived from (18) can be expressed a real heat conduction. Figure 9 (b) shows the transient responses of temperature value in case of exp. A. These experimental results were compared to the simulation values that are derived from the theoretical values at selected point of virtual heat-conduction material. From Fig. 9 (b), temperature responses are almost same as the simulation results. Therefore, it is found that temperature responses can be controlled to the thermal model based on a thermal diffusion equation. The bode diagram between the heat flow response of the device 1 and the heat flow command of device 2 is shown in Fig. 10. From Fig. 10, the frequency characteristics are almost same with the theoretical value within the bandwidth between $10^{-2} \mathrm{rad} / \mathrm{s}$ and $10^{2} \mathrm{rad} / \mathrm{s}$. Therefore, the thermal model derived from Section 2.3 can be implemented in the experiments.

\subsubsection{Verification of Heat-Conduction Control}

Figures 11 (a) and (b) show the temperature and heat flow responses in case of exp. B-1, respectively. From Fig. 11 (a), the transient responses and the attainment temperature of each cases are different and these experimental results of temperature responses were almost same with the theoretical values. In particular, the wider the position between the boundary heat sources, the slower heat conducts. This is because the model of virtual heat conduction is changed depending on the position on the heat-conduction material. From Fig. 11 (b), the command value of heat flow becomes large depending on the rendered position. Therefore, the heat flow command is changed depending on rendered position, and temperature responses are changed accordingly. In this experiments, the heat conduction is slow because of using the theoretical value of copper wire that the length is $45 \mathrm{~mm}$. Conduction speed can become faster if the thermal diffusivity

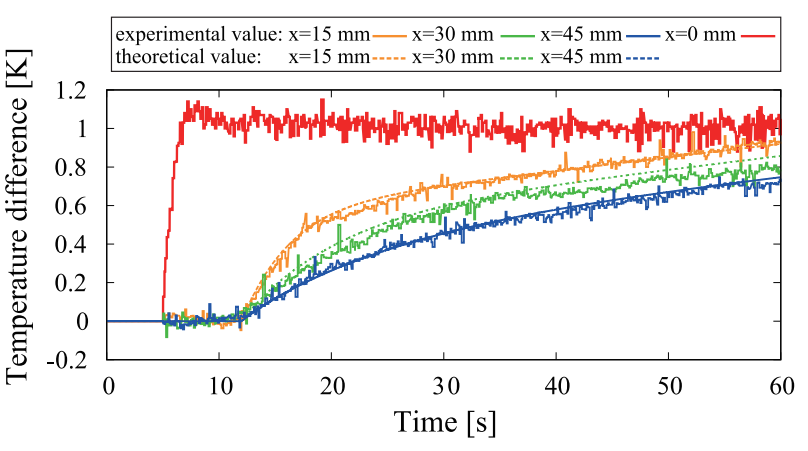

(a) Experimental results of temperature responses.

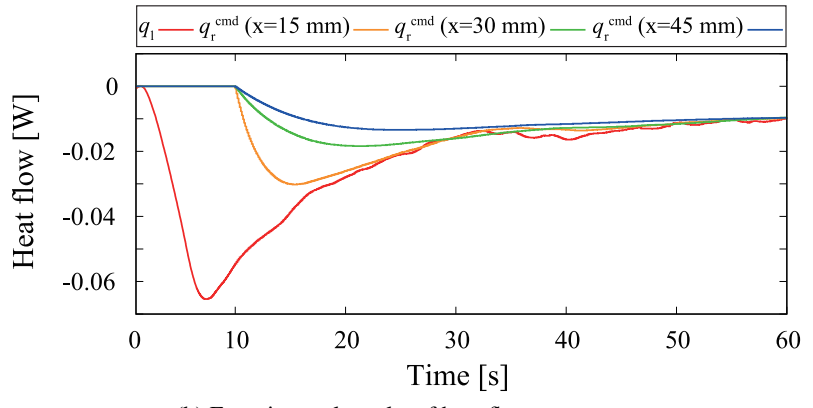

(b) Experimental results of heat flow responses.

Fig. 11. Experimental results of exp. B-1

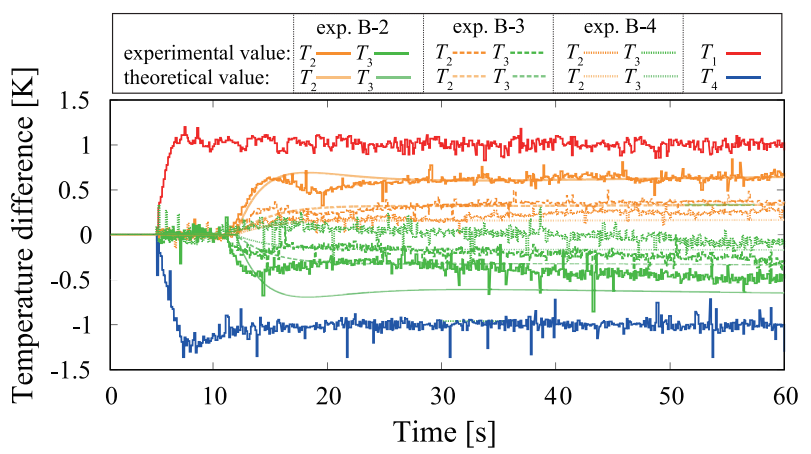

(a) Experimental results of temperature responses.

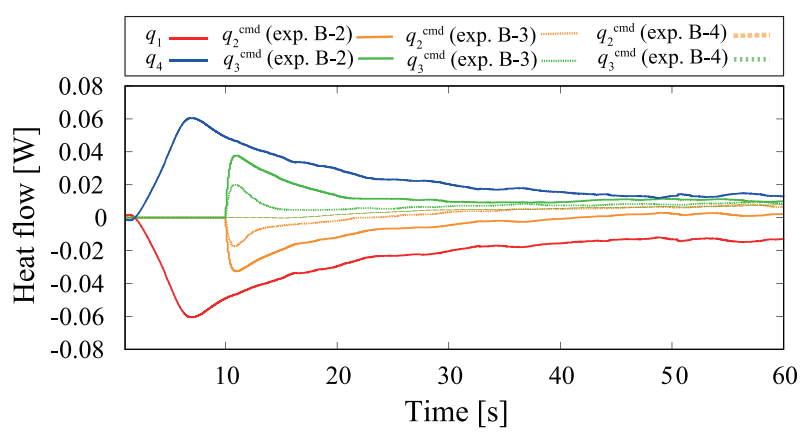

(b) Experimental results of heat flow command.

Fig. 12. Experimental results of exps. B-2, B-3, and B-4

is set to the larger value. Figures 12 (a) and (b) show the temperature and heat flow responses in case of exps. B-2, B-3, and B-4, respectively. From Figs. 12 (a) and (b), selected point from Fig. 8 can be rendered. There is a tendency that heat flow command is effected from closer boundary heat source. From these results, freely-selected point of heat conduction can be rendered using a limited number of heat sources. 


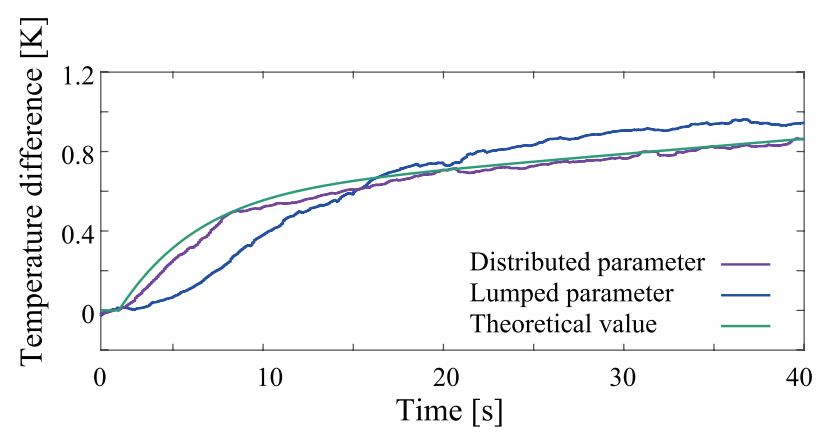

Fig. 13. Experimental results of exp. C

\subsubsection{Comparing to Lumped Parameter Model}

Figure 13 shows temperature responses of exp. C comparing to virtual conduction derived from a lumped parameter model. From Fig. 13, transient response based on a distributed parameter model is close in theoretical value. However, there is an error between response based on a lumped parameter model and the theoretical value. The reason of the error is that the lumped parameter model did not include all the order of the heat conduction model derived from a thermal diffusion equation. In the case of changing location of the rendering point using the conventional method, the number of the thermal capacitances and the thermal parameters have to be changed. Thus, it is difficult to render a transient response of temperature at selected point using a lumped parameter model, despite complicated calculation of the thermal parameter is required. By using the proposed method, selected point of heat-conduction material can be rendered easily. Therefore, the validity of the proposed method can be verified from some experimental results.

\section{Conclusions}

In this paper, the method that model derived from a thermal diffusion equation is used between two heat sources was proposed. By using the proposed method, freely-selected point of the heat conduction can be expressed by only one heat source. Therefore, infinity nodes of heat conduction can be reproduced by selecting a desired node. The validity of the method was verified by some experimental results. The proposed method was derived from one-dimensional thermal diffusion equation and it is limited the applicable range. However, the method is expected to extend to two-dimensional plane by integrating the methods or using two-dimensional thermal diffusion equation. These methods will be considered in the future works. Therefore, the proposed method in this paper is the first step of these multidimensional methods, and it can be a fundamental theory for extending to rendering area of thermal sensation. The method is expected to extend the technology for controlling multiple actuators or heat sources on a surface.

Acknowledgment

This work was partially supported by JSPS KAKENHI $18 \mathrm{H} 03784$.

\section{References}

(1) A. Israr and I. Poupyrev: "Control Space of Apparent Haptic Motion", Proc. VRST (2016)

( 2 ) A. Israr, Z. Schwemler, J. Mars, and B. Krainer: "VR360HD: A VR360" Player with Enhanced Haptic Feedback", Proc. IEEE WHC, pp.457-462 (2011)

( 3 ) G.J. Monkman and P.M. Taylor: "Thermal Tactile Sensing", IEEE Trans. RA, Vol.9, No.3, pp.313-318 (1993)

( 4 ) M.B. Khoudja, M. Hafez, J.M. Alexandre, J. Benachour, and A. Kheddar: "Thermal Feedback Model for Virtual Reality", Proc. IEEE MHS, pp.153158 (2003)

( 5 ) A. Yamamoto, B. Cros, H. Hashimoto, and T. Higuchi: "Thermal Tactile Presentation with On-Site Parameter Identification of Finger", Proc. IEEE ISIE, pp.1365-1370 (2005)

( 6 ) H. Morimitsu and S. Katsura: "Two-Degree-of-Freedom Robust Temperature Control of Peltier Device Based on Heat Disturbance Observer", IEEJ Trans. IA, Vol.13, No.7, pp.967-973 (2011)

( 7 ) M. Guitani, A. Benallegue, and A. Kheddar: "Learning-Based Thermal Rendering in Telepresence", Lecture Notes in Computer Science, Vol.5024, pp.820-825 (2008)

( 8 ) A. Drif, J. Citérin, and A. Kheddar: "Thermal Bilateral Coupling in Teleoperators", Proc. IEEE/RSJ IROS, pp.1301-1306 (2005)

(9) M. Guitani and A. Kheddar: "Modeling Identification and Control of Peltier Thermoelectic Modules for Telepresence", ASME J. Dyn. Syst. Meas. Control, Vol.133, No.3, pp.1-8 (2011)

(10) Y. Osawa, H. Morimitsu, and S. Katsura: "Control of Thermal Conductance with Detection of Single Contacting Part for Rendering Thermal Sensation", IEEJ J. Ind. Appl., Vol.5, No.2, pp.101-107 (2016)

(11) S. Gallo, L.S. Carreras, G. Rognini, M. Hara, A. Yamamoto, and T. Higuchi: "Towards Mutimodal Haptics for Teleoperation: Design of a Tactile Display", Proc. IEEE AMC, pp.1-5 (2012)

(12) M. Zhipeng, W. Jianfeng, L. Jianging, Z. Lianjie, L. Xiaomin, and Y. Yurong: "A Thermal Tactile Display Device with Multiple Heat Sources", Proc. IEEE ICEE, pp.192-195 (2012)

(13) A. Manasrah, N. Crane, R. Guldiken, and K.B. Reed: "Perceived Cooling Using Asymmetrically-Applied Hot and Cold Stimuli", IEEE Trans. HAPTICS, Vol.10, No.1, pp.75-83 (2017)

(14) A. Singhal and L.A. Jones: "Space-time Interaction and the Perceived Location of Cold Stimuli", Proc. IEEE HAPTICS, pp.92-97 (2016)

(15) S. Wen, G. Zhang, Y. Dan, D. Wang, and M. Deng: "Model Output Following Control for An Aluminum Plate Cooling Process with a Peltier Device", Proc. IEEE ICAMechS, Vol.11, No.2, pp.452-457 (2012)

(16) Y. Osawa and S. Katsura: "Distributed Thermal Conductance Control for Sensing and Rendering Thermal Sensation on the Palm", Proc. IEEE AMC, pp.328-333 (2018)

(17) I.S. Jesus, J.A.T. Machado, and R.S. Barbosa: "Towards the PID $\beta$ Control of Heat Diffusion Systems", Proc. IEEE ISDA, pp.913-918 (2007)

(18) G. Ichimasa, K. Okumura, H. Okajima, and N. Matsunaga: "Extended Structure of MEC for Thermal Process", Proc. SICE Annu. Conf., pp.1593-1598 (2016)

(19) Y. Osawa and S. Katsura: "Thermal Propagation Control Using Thermal Diffusion Equation", IEEE Trans. Ind. Electron., Vol.65, No.11, pp.8809-8817 (2018)

(20) A. Sarikhani and O.A. Mohammed: "Inter-Turn Fault Detection in PM Synchronous Machines by Physics-Based Back Electromotive Force Estimation”, IEEE Trans. Ind. Electron., Vol.60, No.8, pp.3472-3484 (2013)

(21) A.A. Hasmasan, C. Busca, R. Teodorescu, L. Helle, and F. Blaabjerg: "Electro-Thermo-Mechanical Analysis of High-Power Press-Pack Insulated Gate Bipolar Transistors under Various Mechanical Clamping Conditions", IEEJ J. Ind. Appl., Vol.3, No.3, pp.192-197 (2014)

(22) M. Macias and D. Sierociuk: "Fractional Order Calculus for Modeling and Fractional PID Control of the Heating Process", Proc. IEEE ICCC, pp.453457 (2012)

(23) C.A. Monje, Y. Chen, B.M. Vinagre, D. Xue, and V. Felu: "Fractional-order Systems and Controls: Fundamentals and Applications", Springer Science \& Business Media (2010) 
Yukiko Osawa (Member) received her B.E. degree in system design

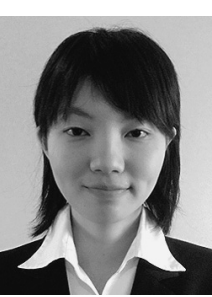
engineering and the M.E. and Ph.D. degrees in integrated design engineering from Keio University, Yokohama, Japan, in 2015, 2016 and 2019, respectively. From 2017 to 2019, she was a Research Fellow of the Japan Society for the Promotion of Science (JSPS). From 2019, she is a JSPS Overseas Research Fellow. Her research interests include thermotics, distributed parameter systems, human support space, and human interface. She is a Member of IEEJ, as well as IEEE. She was the recipient of the IEEJ Distinguished Paper Award in 2017 and JSPS Ikushi Prize in 2019.
Seiichiro Katsura (Senior Member) received the B.E. degree in sys-

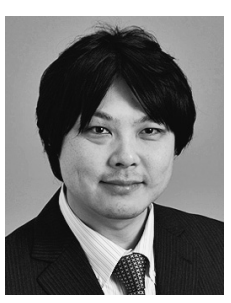
tem design engineering and the M.E. and Ph.D. degrees in integrated design engineering from Keio University, Yokohama, Japan, in 2001, 2002 and 2004, respectively. From 2003 to 2005, he was a Research Fellow of the Japan Society for the Promotion of Science (JSPS). From 2005 to 2008, he worked at Nagaoka University of Technology, Nagaoka, Niigata, Japan. Since 2008, he has been at Department of System Design Engineering, Keio University, Yokohama, Japan. Currently he is working as Professor. In 2017, he was a Visiting Researcher with the Laboratory for Machine Tools and Production Engineering (WZL) of RWTH Aachen University, Aachen, Germany. His research interests include applied abstraction, human support, data robotics, wave system, systems energy conversion, and electromechanical integration systems. Prof. Katsura serves as an Associate Editor of the IEEE Transactions on Industrial Electronics and Technical Editor of IEEE/ASME Transactions on Mechatronics. He was the recipient of IEEJ Distinguished Paper Awards in 2003 and 2017, IEEE Industrial Electronics Society Best Conference Paper Award in 2012, and JSPS Prize in 2016. 